\title{
Peri-operative risk factors for in-hospital mortality in acute type A aortic dissection
}

\author{
Miaoyun Wen ${ }^{1 \#}$, Yongli Han ${ }^{1 \#}$, Jingkun Ye ${ }^{2 \#}$, Gengxin Cai ${ }^{3}$, Wenxin Zeng ${ }^{1}$, Xinqiang Liu ${ }^{1}$, Linqiang Huang ${ }^{1}$ \\ Zhesi Lian ${ }^{4}$, Hongke Zeng ${ }^{1}$ \\ ${ }^{1}$ Department of Intensive Care Unit 1, Guangdong General Hospital, Guangdong Academy of Medical Sciences, Guangzhou 510080, China; \\ ${ }^{2}$ Zhujiang Hospital, Southern Medical University, Guangzhou 510280, China; ${ }^{3}$ School of Medicine, South China University of Technology, \\ Guangzhou 510641, China; ${ }^{4}$ Department of Public Health, Tufts University, School of Medicine, Boston, MA, USA \\ Contributions: (I) Conception and design: M Wen, Y Han, J Ye, Z Lian, H Zeng; (II) Administrative support: G Cai, W Zeng, X Liu, L Huang; (III) \\ Provision of study materials or patients: M Wen, Y Han, J Ye; (IV) Collection and assembly of data: M Wen, G Cai, W Zeng, X Liu, L Huang; (V) \\ Data analysis and interpretation: M Wen, Y Han, J Ye, Z Lian; (VI) Manuscript writing: All authors; (VII) Final approval of manuscript: All authors. \\ \#These authors contributed equally to this work. \\ Correspondence to: Zhesi Lian. 136 Harrison Avenue, Boston, MA 02111, USA. Email: zhesi.lian@tufts.edu.
}

\begin{abstract}
Background: Acute type A aortic dissection (TAAD) is cardiovascular emergency and requires surgical interventions. In-hospital mortality rate of surgical-treated TAAD patients remains high. We aim to examine the prognostic implications of peri-operative parameters to identify high-risk patient for in-hospital mortality.
\end{abstract}

Methods: A total of 264 surgically treated TAAD patients were included in this study. The association between in-hospital mortality and peri-operative parameters were examined.

Results: Thirty patients (11.36\%) died during hospitalization. Patients with higher Apache II score had a significantly higher rate of in-hospital mortality when compared with patients scored $\leq 20$ in unadjusted model [Score 21-25: HR =12.9 (1.7-100.8), $\mathrm{P}=0.0148$; Score $>25$ : HR =94.5 (12.6-707.6), $\mathrm{P}<0.0001$ ]. Patients with $\mathrm{Sbp}>120 \mathrm{mmHg}, \mathrm{Cr}>200 \mathrm{mmol} / \mathrm{L}$ (both at admission and after surgery), BUN $>8.2$ mmol/L (both at admission and after surgery), AST $>80 \mu / \mathrm{L}$, aortic cross-clamping time $>120 \mathrm{~min}$ and cardiopulmonary bypass time (CPBT) >230 min were also significantly related to higher rate of in-hospital mortality in univariate analysis. In multivariable analysis, APACHE II score [Score 21-25: HR =9.5 (1.2-74.4), $\mathrm{P}=0.032$; Score $>25: \mathrm{HR}=51.0$ (6.7-387.7), $\mathrm{P}=0.0001]$, AST >80 $\mu \mathrm{mol} / \mathrm{L}$ [HR =2.3 (1.1-4.8), $\mathrm{P}=0.0251]$, aortic cross-clamping time $>120 \mathrm{~min}(\mathrm{HR}=2.9(1.1-7.7), \mathrm{P}=0.0315)$ remained significant in predicting TAAD in-hospital mortality.

Conclusions: APACHE II score could be a useful tool to predict TAAD in-hospital mortality. AST $>80 \mu / \mathrm{L}$ and aortic cross-clamping time $>120 \mathrm{~min}$ were also independent predictors.

Keywords: Acute type A aortic dissection (TAAD); in-hospital mortality; APACHE II score; peri-operative risk factor

Submitted Jun 11, 2019. Accepted for publication Aug 23, 2019.

doi: $10.21037 /$ jtd.2019.09.11

View this article at: http://dx.doi.org/10.21037/jtd.2019.09.11

\section{Introduction}

Acute type A aortic dissection (TAAD) is cardiovascular emergency that is often fatal. American Heart Association guidelines recommend that surgical interventions should be given to TAAD patients to resect all aneurysmal aorta and the proximal extent of the dissection (1). TAAD surgical managements had been increased and improved in recent years. In 2015, international registry of acute aortic dissection (IRAD) reported that in-hospital mortality of surgical treated TAAD patients has decreased over time, but the rate remained high (from $25 \%$ to $18 \%$ over 
17 years, $\mathrm{P}=0.003$ ) (2). Identifying patients with high-risk feature is clinically relevant. IRAD researchers reported that the principal independent pre-operative predictors of mortality were history of aortic valve replacement, migrating chest pain, hypotension as sign of TAAD, shock or tamponade, preoperative cardiac tamponade, and preoperative limb ischemia (3). Age greater than 70 years, prior cardiac surgery, intraoperative hypotension, a right ventricle dysfunction at surgery, a necessity to perform coronary revascularization, pre-existing cardiac disease, and cardiopulmonary resuscitation were also reported as risk factors for TAAD in-hospital mortality in other studies $(4,5)$.

Since TAAD is an acute condition and often progresses rapidly, it could be hard to gather all the relevant data during hospitalization. Studies were lacking to explore potential prognostic values of biomarkers, traditional disease severity measurement system (e.g., APACHE II score), and intra-operative parameters. In the current study, we aim to examine the prognostic implications of such parameters to identify high-risk patient for in-hospital mortality.

\section{Methods}

\section{Study design and patients' selection}

This is a retrospective analysis of consecutive TAAD patients in Guangdong General Hospital from March 2012 to April 2016. All patient data were collected by chart review. Patients who died before and during surgery were excluded ( $n=4)$, and a total of 264 patients were analyzed. This retrospective study did not require ethics approval.

\section{Measurement and outcome}

Diagnosis of TAAD was by contrast-enhanced computed tomography scan and identified by dissections involving the ascending aorta. The primary endpoint of the study is in-hospital mortality, which is defined as death within first TAAD related hospitalization during which surgical treatment was rendered. In-hospital mortality in this study included 30-day mortality and after 30-day mortality (but in-hospital mortality). Pre-operative parameters [age, sex, history of hypertension, APACHEII score, systolic blood pressure (Sbp), diastolic blood pressure (Dbp), mean arterial pressure (MAP), temperature, heart rate (HR), hemoglobin count (Hbg), white blood cell count (WBC), albumin level (Alb), blood creatinine level (Cr), blood urea nitrogen level (BUN), alanine aminotransferase level (Alt), aspartate transaminase(AST), serum total bilirubin (TBil), direct bilirubin (DBil)], intra-operative parameters [aortic crossclamping time (XCT) and cardiopulmonary bypass time (CPBT)], and post-operative parameters ( $\mathrm{Cr}$ immediate after surgery, BUN immediate after surgery, ICU stay) were prospectively entered in electronic health medical system and retrospectively collected. Pre-operative parameters were recorded by physicians and nurses at admission. APACHE II score was calculated by emergency room physicians. XCT and CPBT were recorded by perfusionist during surgery.

\section{Statistical analysis}

Categorical variables and continuous variables are analyzed with Chi-square test and $t$-test respectively. Cox proportional hazard model was used for univariate and multivariable regression. Survival was estimated using Kaplan-Meier curve (and compared using log-rank test). A two-sided $\mathrm{P}$ value $<0.05$ was considered statistically significant. SAS 9.4 was used to perform all statistical analysis.

\section{Results}

\section{Patients' characteristics}

From March 2012 to April 2016, a total of 264 TAAD patients were surgically treated in Guangdong General Hospital. The demographic and disease characteristics were summarized in Table 1. Mean hospitalization duration was $31.96 \pm 19.26$ days. Reasons of long hospital stay (more than 30 days) included $(n=100)$ : infection $(n=63)$, impaired wound healing $(n=9)$, kidney failure $(n=15)$, respiratory failure $(n=6)$, liver failure $(n=6)$, heart failure $(n=5)$, pleural effusion ( $n=3)$, pericardial effusion $(n=3)$, post-operation bleeding $(n=3)$, stroke $(n=3)$, gastrointestinal bleeding $(\mathrm{n}=2)$, unconsciousness $(\mathrm{n}=2)$, vocal cord injury $(\mathrm{n}=2)$, renal arterial thrombosis $(n=1)$, nerve injury $(n=1)$, and paraplegia $(\mathrm{n}=1)$. Patients who did not survive during hospitalization had an average higher APACHE II score $(\mathrm{P}<0.0001)$, WBC $(\mathrm{P}=0.0006)$, BUN $(\mathrm{P}=0.0274)$, longer XCT $(\mathrm{P}=0.0002)$ and CPBT $(\mathrm{P}<0.0001)$. Thirty patients $(11.36 \%)$ died during hospitalization: 7 from septic shock (23.33\%), 5 from malignant arrhythmia (16.67\%), 5 from brain death (16.67\%), 3 from low cardiac output (10.00\%), 3 from severe pneumonia (10.00\%), 2 from gastrointestinal hemorrhage $(6.67 \%), 1$ from aortic dissection rupture $(3.33 \%), 1$ from cardiac tamponade $(3.33 \%), 1$ from hematogenic shock (3.33\%), 1 from acute kidney failure 
Table 1 Patients' characteristics

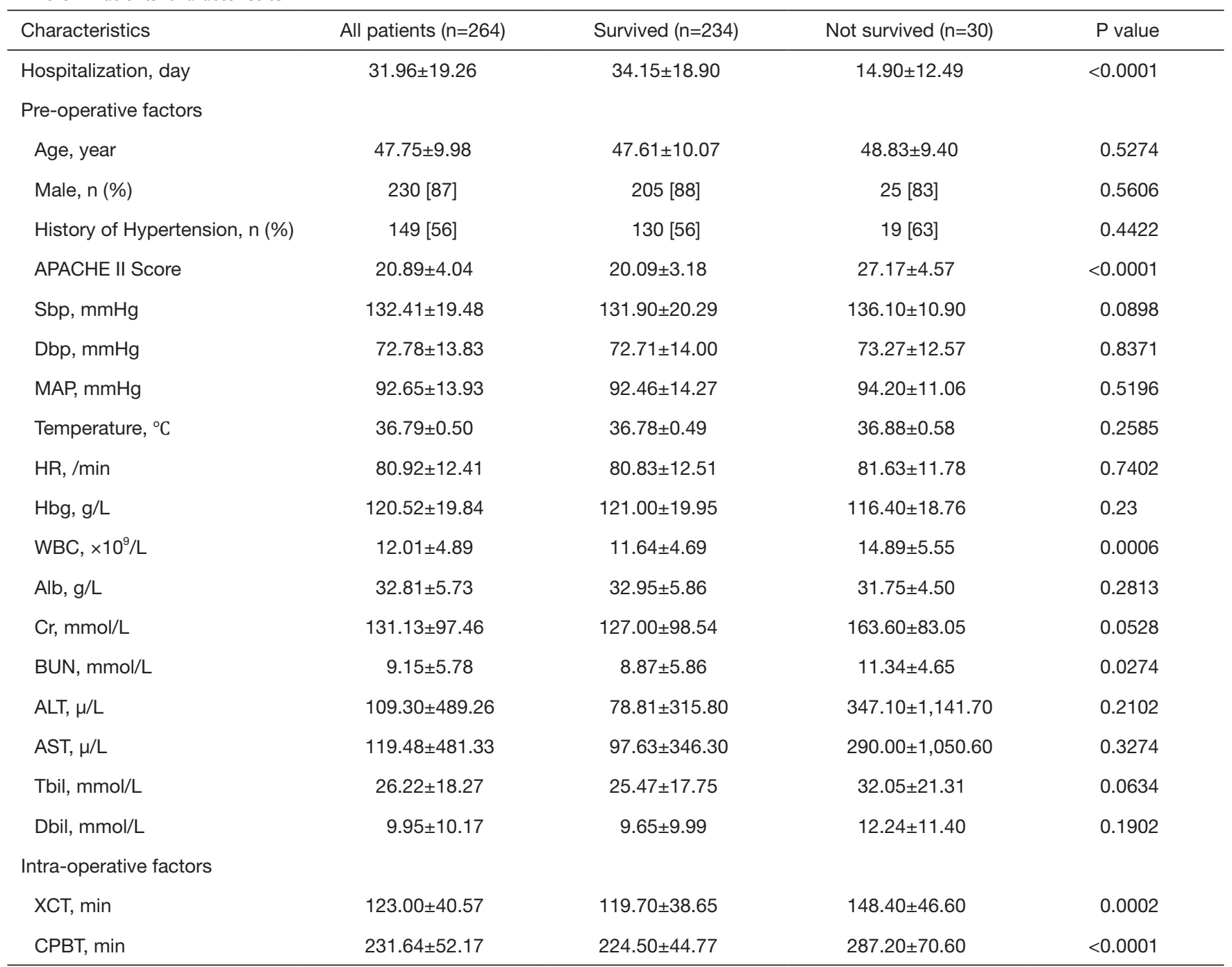

Values are mean \pm SD or $\mathrm{n}(\%)$. Alb, albumin level; ALT, alanine aminotransferase level; AST, aspartate transaminase; BUN, blood urea nitrogen level; CPBT, cardiopulmonary bypass time; Cr, blood creatinine level; DBil, direct bilirubin; Dbp, diastolic blood pressure; Hbg, hemoglobin count; HR, heart rate; MAP, mean arterial pressure; Sbp, systolic blood pressure; TBil, serum total bilirubin; WBC, white blood cell count; $\mathrm{XCT}$, aortic cross-clamping time.

(3.33\%), 1 from alveolar hemorrhage (3.33\%).

\section{APACHE II score and in-bospital mortality}

The range of APACHE II score of these patients was 13 to 40. Table 2 showed the percentage of death by APACHE II score. There was an abrupt increase in in-hospital mortality when APACHE II score was more than 25. Figure 1 showed that the in-hospital survival rate of all the patients was $82.86 \%$. When stratifying by APACHE II score, patients with score higher than 25 had a significant lower in-hospital survival $(35.67 \%)$ then patient with lower score (score $<20$ : $99.1 \% ; 21-25: 86.76 \%, \mathrm{P}<0.0001)$

Table 3 showed that, compared with patients with score $\leq 20$, patients with higher score had a significantly higher rate of in-hospital mortality in unadjusted model [21-25: HR $=12.9(1.7-100.8), \mathrm{P}=0.0148 ;$ Score $>25: \mathrm{HR}=94.5$ (12.6-707.6), $\mathrm{P}<0.0001]$.

\section{Univariate analysis}

Besides APACHE II score, SBP >120 mmHg, Cr 
Table 2 APACHE II score and mortality distribution

\begin{tabular}{lccc}
\hline APACHE II score & Total & Death & Percentage \\
\hline $0-15$ & 11 & 0 & 0.0 \\
$16-20$ & 117 & 1 & 0.9 \\
$21-25$ & 104 & 10 & 9.6 \\
$26-30$ & 24 & 13 & 54.2 \\
$31-35$ & 6 & 4 & 66.7 \\
$36-40$ & 2 & 2 & 100.0 \\
Total & 264 & 30 & 11.4 \\
\hline
\end{tabular}

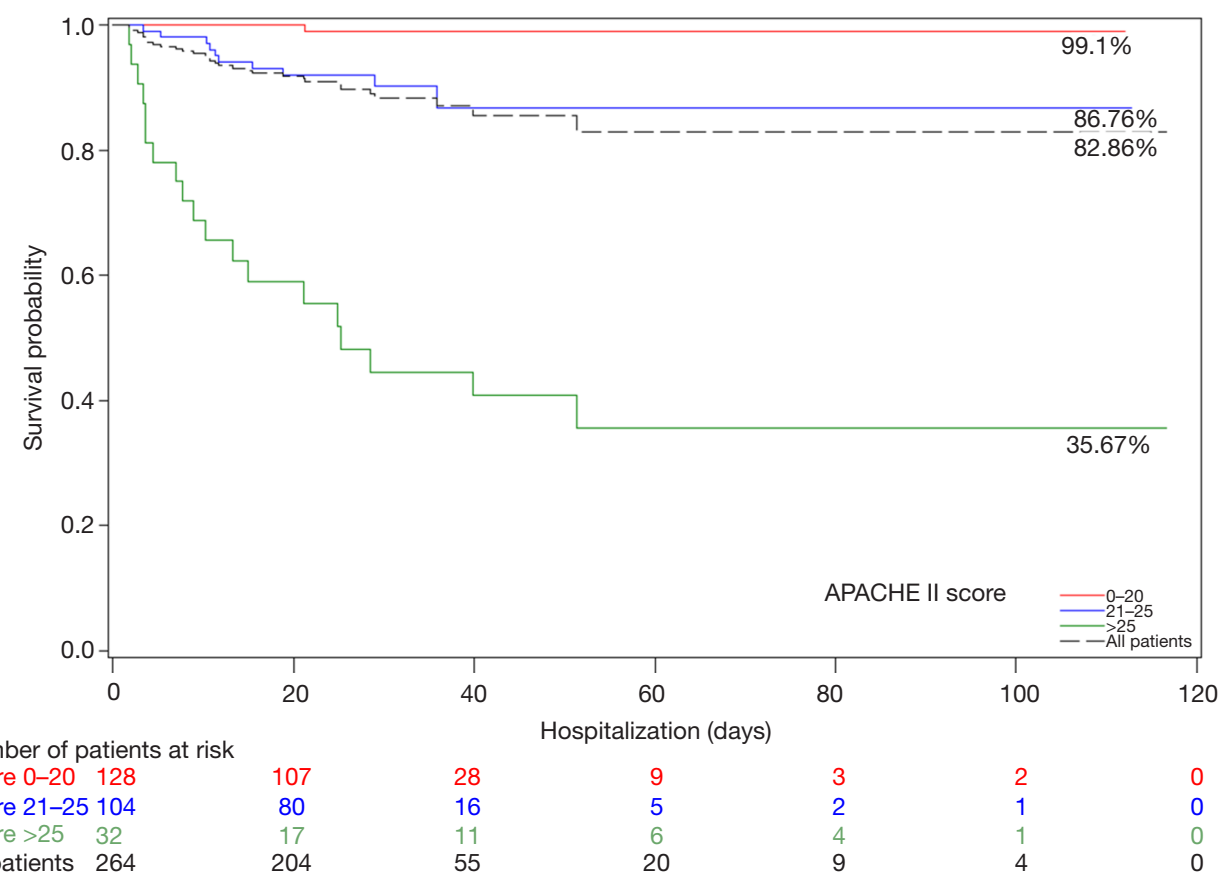

Figure 1 Surgical-treated type A aortic dissection patients' in-hospital survival probability by APACHE II score.

$>200 \mathrm{mmol} / \mathrm{L}$ (both at admission and after surgery), BUN $>8.2 \mathrm{mmol} / \mathrm{L}$ (both at admission and after surgery), AST $>80 \mu / \mathrm{L}, \mathrm{XCT}>120 \mathrm{~min}$ and $\mathrm{CPBT}>230 \mathrm{~min}$ were also significantly associated with higher rate of in-hospital mortality in univariable analysis (Table 3).

\section{Multivariable analysis}

In multivariable analysis (Table 4), using variables which were available and related to APACHE II score (age, temperature, $\mathrm{HR}, \mathrm{Cr}$ at admission, MAP) and other important factors (Sbp, AST and aortic cross-clamping time) (model 1), we found that $\mathrm{SBP}>120 \mathrm{mmHg}$, AST $>80 \mu / \mathrm{L}$, and XCT >120 min were independent predictors of in-hospital mortality.

After adjusting for APACHE II score (model 2), AST $>80 \mu / \mathrm{L}$ and XCT $>120$ min remained significant.

\section{Discussion}

TAAD is a cardiovascular emergency that requires prompt surgical intervention. Despite advances in surgical technique, the disease is still fatal, with in-hospital mortality reported between $10.6 \%$ to $26.6 \%(3,5-10)$. Identifying 
Table 3 Univariate analysis

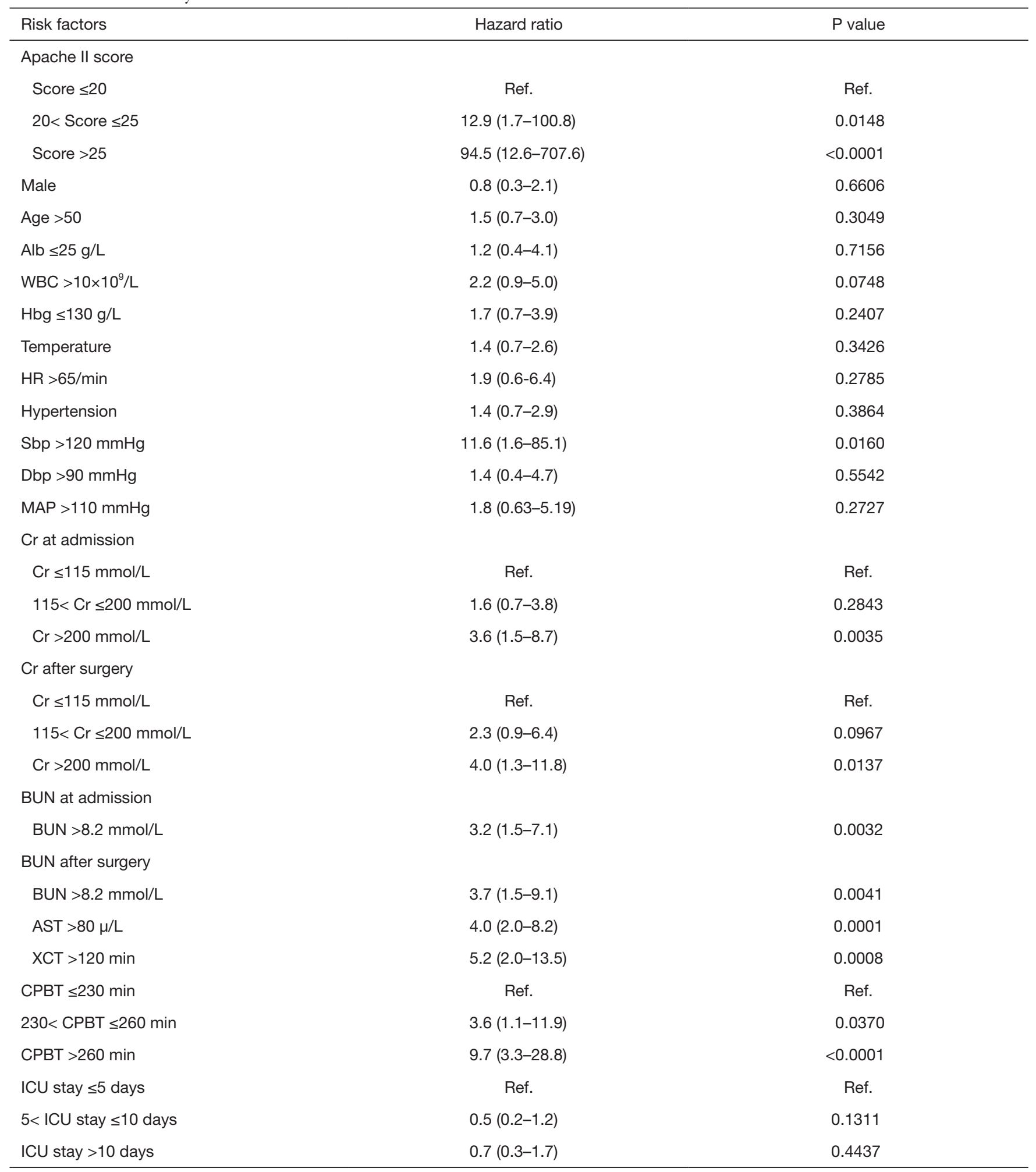

Alb, albumin level; AST, aspartate transaminase; BUN, blood urea nitrogen level; CPBT, cardiopulmonary bypass time; Cr, blood creatinine level; Dbp, diastolic blood pressure; Hbg, hemoglobin count; HR, heart rate; MAP, mean arterial pressure; Sbp, systolic blood pressure; WBC, white blood cell count; XCT, aortic cross-clamping time. 
Table 4 Multivariable analysis

\begin{tabular}{|c|c|c|}
\hline Risk factors & Hazard ratio & $P$ value \\
\hline \multicolumn{3}{|l|}{ Model 1} \\
\hline Age $>50$ & $1.9(0.9-4.1)$ & 0.0995 \\
\hline Temperature & $1.5(0.7-3.2)$ & 0.289 \\
\hline $\mathrm{HR}>65 / \mathrm{min}$ & $1.2(0.4-4.2)$ & 0.7398 \\
\hline $115<\mathrm{Cr} \leq 200 \mathrm{mmol} / \mathrm{L}$ & $1.3(0.5-3.1)$ & 0.5834 \\
\hline $\mathrm{Cr}>200 \mathrm{mmol} / \mathrm{L}$ & $1.8(0.7-4.8)$ & 0.2442 \\
\hline WBC $>10 \times 10^{\wedge} 9 / L$ & $1.1(0.4-2.7)$ & 0.8885 \\
\hline MAP > $110 \mathrm{mmHg}$ & $1.2(0.4-3.5)$ & 0.7616 \\
\hline XCT >120 min & $4.2(1.5-11.2)$ & 0.0048 \\
\hline \multicolumn{3}{|l|}{ Model 2} \\
\hline APACHE II Score $\leq 20$ & Ref. & Ref. \\
\hline $20<$ Score $\leq 25$ & $9.5(1.2-74.4)$ & 0.032 \\
\hline Score $>25$ & $51.0(6.7-387.7)$ & 0.0001 \\
\hline $\mathrm{SBP}>120 \mathrm{mmHg}$ & $6.5(0.9-48.1)$ & 0.0668 \\
\hline AST $>80 \mu / L$ & $2.3(1.1-4.8)$ & 0.0251 \\
\hline $\mathrm{XCT}>120 \mathrm{~min}$ & $2.9(1.1-7.7)$ & 0.0315 \\
\hline
\end{tabular}

${ }^{\dagger}, \mathrm{Cr}$ at admission. AST, aspartate transaminase; CPBT, cardiopulmonary bypass time; Cr, blood creatinine level; HR, heart rate; MAP, mean arterial pressure; Sbp, systolic blood pressure; WBC, white blood cell count; XCT, aortic cross-clamping time.

high risk patients is thus clinically relevant as clinical trials may target those patients for innovation in surgical techniques and peri-operative management.

In the current study, we analyzed the outcome of 264 TAAD patients who received surgical treatment in Guangdong General Hospital. The in-hospital mortality was $11.4 \%$. This was consistent with study by Masuda and colleagues (10.6\% in 2014) (6), and was lower than those from other investigators (3,5,7-10). This may reflect continuous improvement in surgical technique and perioperative management. However, race and referral-based facility may also explain the relatively low mortality. We found that APACHE II score could be a useful tool to predict in-hospital mortality. AST $>80 \mu / \mathrm{L}$ and XCT $>120$ min were also independent predictors.

APACHE II score is a severity of disease classification system based on 12 physiologic measurements, age and previous health status (11). It is a well validated score system that is widely used in ICU setting. Due to its limitation, it may not be appropriate for all disease types. Giamarellos-Bourboulis et al. discussed that if comparing patients at a wide range of age with various baseline condition (e.g., septic patients), APACHE II score may not be sufficient to estimate death risk (12). There was no previous study investigating whether APACHE II score is suitable for predicting TAAD mortality. In current study, the mortality rate increased significantly as the score increased. In our cohort, most patients were healthy nonelderly adults with few comorbidities. APACHE II score provided a good prediction for TAAD in-hospital mortality. We also calculated the area under the receiver operating characteristics curve (AUROC) for APACHE II score (Figure S1). The AUROC was $0.9172(\mathrm{P}<0.0001)$, which also proved that APACHE II score is an excellent model to predict TAAD in-hospital death. APACHE II score could quantify severity and help physicians to have a general idea 
about patients' situation at admission. The score system may also be used in future TAAD studies to identify high risk patients. We originally planned to decompose the score system to analyze each variable, but we were only able to collect age, temperature, heart rate, $\mathrm{Cr}$ level, WBC counts and MAP data. We analyzed the available variables and found that only Cr level greater than $200 \mu \mathrm{mol} / \mathrm{L}$ was related to TAAD in-hospital mortality in univariate analysis, but all of them were not significant in adjusted model. This might indicate that the other variables in the score system $\left(\mathrm{AaDO}_{2}\right.$ or $\mathrm{PaO}_{2}, \mathrm{pH}$ arterial, respiratory rate, serum sodium, serum potassium, Glasgow coma scale) could have a greater weight in predicting TAAD in-hospital mortality. The prognostic implication of APACHE II score in TAAD patient will need to be further confirmed in future studies.

Among other pre-operative biomarkers, AST $>80 \mu / \mathrm{L}$ was an independent predictor besides APACHE II score. One study found that elevated AST levels was significant in univariate analysis but marginally insignificant in multivariable analysis $(\mathrm{OR}=3.204$; 95\% CI, 0.986-10.409, $\mathrm{P}=0.0527)$ (13). A high AST level may result from organ injury (liver, heart, muscle, etc.) and/or inflammatory response to acute stress. Abnormal baseline AST may also indicate pre-existing liver disease, or organ ischemia during TAAD. Another research showed that TAAD patients' AST level also tended to increase after surgery (14). Because gastrointestinal complications (15), kidney injury (16), myocardial ischemia are frequently present during or after TAAD surgeries, patients with abnormal pre-operative AST level at baseline might have a greater organ injury or inflammatory response during and after surgery, which may be associated with higher mortality. This result may also suggest that liver protection and anti-inflammatory treatment should be further investigated in patients treated surgically.

TAAD surgeries are technically challenging and require extra-corporal cardiopulmonary bypass. We found that patients whose XCT more than 2 hours had over 4 times higher risk of in-hospital mortality. It is not new that long XCT is related to patients' death in general heart surgeries, but such data are lacking in TAAD surgeries. Nissinen et al. found that XCT $>150 \mathrm{~min}$ was associated with a high risk of 30 -day mortality $(\mathrm{OR}=1.21 / 30 \mathrm{~min}$, 95\% CI, 1.01-1.52) independently of the complexity of surgery (17). Doenst et al. also found that XCT was an independent predictor of mortality for patients with LVEF $>40 \%$ regardless of surgery complexity $(\mathrm{OR}=1.014$ per $\mathrm{min}$ of XCL, 95\% CI, 1.01-1.02) (18). Regarding TAAD, Goda et al. found that TAAD nonsurvivors had much longer XCT than survivors $(206 \pm 96$ vs. $161 \pm 58 \mathrm{~min})(13)$. Some researchers had also found that long CPBT was related to mortality (19-21). In our study, CPBT was a significant risk factor in univariate analysis, but not significant after adjusted for APACHE II score and AST (230-260 vs. $<230 \mathrm{~min}, \mathrm{HR}=2.193,95 \%$ CI: $0.654-7.353, \mathrm{P}=0.2034$; $>260$ vs. $<230 \mathrm{~min}, \mathrm{HR}=3.127,95 \% \mathrm{CI}, 0.997-9.812$, $\mathrm{P}=0.0507)$. XCT remained significant in adjusted models. $\mathrm{XCT}$ and CPBT were highly correlated in our data (correlation coefficients $=0.74332, \mathrm{P}<0.0001$ ), and XCT length is the determinant factor of CPBT length. We believe that they are similar important predictors, but XCT might have slightly better predicting value. A prolonged XCT indicates technical challenge and/or complications during surgery, which might affect patient outcome, such as longer ICU stay (22) and TAAD postoperative renal impairment and renal failure (23). These factors might then affect patents' in-hospital mortality. Therefore, improving intra-operative management and shorten XCT is an important way to improve in-hospital mortality.

This study had several limitations. First, this was a single center study and the sample size was relatively small. Less than $5 \%$ of the patient population were older than 65 , which limits generalizability in elderly patients. Second, while prolong CPBT time itself increases mortality, surgery complexity and intraoperative issues might be confounding factors. Third, over-fitting might be a problem when we used Apache II score in disease with low event counts. Since we lacked the data of some important indexes $\left(\mathrm{AaDO}_{2}\right.$ or $\mathrm{PaO}_{2}, \mathrm{pH}$ arterial, respiratory rate, serum sodium, serum potassium, Glasgow coma scale), we were not able to decompose APACHE II score system. We also could not collect the data of the five principle factors discovered by IRAD. Further research with more comprehensive data will be needed to better identify predictors to TAAD in-hospital mortality.

\section{Conclusions}

APACHE II score $>25$, AST $>80 \mu / \mathrm{L}$ and XCT $>120 \mathrm{~min}$ are independent risk factors of surgical treated TAAD patients' in-hospital mortality. Improving peri-operative managements are important to improve patients' outcome.

\section{Acknowledgments}

Funding: This work was supported by the Natural 
Science Foundation of Guangdong Province, China [\#2016A030313763] and Guangzhou Science, Technology and Innovation Commission [\#201707010322].

\section{Footnote}

Conflicts of Interest: The authors have no conflicts of interest to declare.

Ethical Statement: The authors are accountable for all aspects of the work in ensuring that questions related to the accuracy or integrity of any part of the work are appropriately investigated and resolved.

\section{References}

1. Hiratzka LF, Bakris GL, Beckman JA, et al. 2010 ACCF/ AHA/AATS/ACR/ASA/SCA/SCAI/SIR/STS/SVM guidelines for the diagnosis and management of patients with thoracic aortic disease: executive summary. A report of the American College of Cardiology Foundation/ American Heart Association Task Force on Practice Guidelines, American Association for Thoracic Surgery, American College of Radiology, American Stroke Association, Society of Cardiovascular Anesthesiologists, Society for Cardiovascular Angiography and Interventions, Society of Interventional Radiology, Society of Thoracic Surgeons, and Society for Vascular Medicine. Catheter Cardiovasc Interv 2010;76:E43-86.

2. Pape LA, Awais M, Woznicki EM, et al. Presentation, Diagnosis, and Outcomes of Acute Aortic Dissection: 17Year Trends From the International Registry of Acute Aortic Dissection. J Am Coll Cardiol 2015;66:350-8.

3. Trimarchi S, Nienaber CA, Rampoldi V, et al. Contemporary results of surgery in acute type A aortic dissection: The International Registry of Acute Aortic Dissection experience. J Thorac Cardiovasc Surg 2005;129:112-22.

4. Rampoldi V, Trimarchi S, Eagle KA, et al. Simple risk models to predict surgical mortality in acute type A aortic dissection: the International Registry of Acute Aortic Dissection score. Ann Thorac Surg 2007;83:55-61.

5. Chiappini B, Schepens M, Tan E, et al. Early and late outcomes of acute type A aortic dissection: analysis of risk factors in 487 consecutive patients. Eur Heart J 2005;26:180-6.

6. Masuda M, Okumura M, Doki Y, et al. Thoracic and cardiovascular surgery in Japan during 2014 : Annual report by The Japanese Association for Thoracic Surgery. Gen Thorac Cardiovasc Surg 2016;64:665-97.

7. Leontyev S, Légaré JF, Borger MA, et al. Creation of a Scorecard to Predict In-Hospital Death in Patients Undergoing Operations for Acute Type A Aortic Dissection. Ann Thorac Surg 2016;101:1700-6.

8. McClure RS, Brogly SB, Lajkosz K, et al. Epidemiology and management of thoracic aortic dissections and thoracic aortic aneurysms in Ontario, Canada: A population-based study. J Thorac Cardiovasc Surg 2018;155:2254-64.e4.

9. Mehta RH, Suzuki T, Hagan PG, et al. Predicting death in patients with acute type a aortic dissection. Circulation 2002;105:200-6.

10. Santini F, Montalbano G, Casali G, et al. Clinical presentation is the main predictor of in-hospital death for patients with acute type A aortic dissection admitted for surgical treatment: a 25 years experience. Int J Cardiol 2007;115:305-11.

11. Knaus WA, Draper EA, Wagner DP, et al. APACHE II: a severity of disease classification system. Crit Care Med 1985;13:818-29.

12. Giamarellos-Bourboulis EJ, Norrby-Teglund A, Mylona $\mathrm{V}$, et al. Risk assessment in sepsis: a new prognostication rule by APACHE II score and serum soluble urokinase plasminogen activator receptor. Crit Care 2012;16:R149.

13. Goda M, Imoto K, Suzuki S, et al. Risk analysis for hospital mortality in patients with acute type a aortic dissection. Ann Thorac Surg 2010;90:1246-50.

14. Gong $M, M a$ WG, Guan XL, et al. Moderate hypothermic circulatory arrest in total arch repair for acute type A aortic dissection: clinical safety and efficacy. J Thorac Dis 2016;8:925-33.

15. Zheng S, Shao S, Qiao Z, et al. Clinical Parameters and Gut Microbiome Changes Before and After Surgery in Thoracic Aortic Dissection in Patients with Gastrointestinal Complications. Sci Rep 2017;7:15228.

16. Sansone F, Morgante A, Ceresa F, et al. Prognostic Implications of Acute Renal Failure after Surgery for Type A Acute Aortic Dissection. Aorta (Stamford) 2015;3:91-7.

17. Nissinen J, Biancari F, Wistbacka JO, et al. Safe time limits of aortic cross-clamping and cardiopulmonary bypass in adult cardiac surgery. Perfusion 2009;24:297-305.

18. Doenst T, Borger MA, Weisel RD, et al. Relation between aortic cross-clamp time and mortality--not as straightforward as expected. Eur J Cardiothorac Surg 2008;33:660-5.

19. Kawahito K, Adachi H, Yamaguchi A, et al. Preoperative risk factors for hospital mortality in acute type A aortic 
dissection. Ann Thorac Surg 2001;71:1239-43.

20. Biglioli P, Parolari A, Spirito R, et al. Early and late results of ascending aorta surgery: risk factors for early and late outcome. World J Surg 1997;21:590-8.

21. Skripochnik E, Friedman P, Michler RE, et al. The outcome of surgical management of type A aortic dissection. Asian Cardiovasc Thorac Ann 2014;22:687-93.

22. Hoefer D, Ruttmann E, Riha M, et al. Factors influencing intensive care unit length of stay after surgery for acute aortic dissection type A. Ann Thorac Surg 2002;73:714-8; discussion 718-9.

23. Schoenrath F, Laber R, Maralushaj M, et al. Survival, Neurologic Injury, and Kidney Function after Surgery for Acute Type A Aortic Dissection. Thorac Cardiovasc Surg 2016;64:100-7.

Cite this article as: Wen M, Han Y, Ye J, Cai G, Zeng W, Liu $X$, Huang L, Lian Z, Zeng H. Peri-operative risk factors for inhospital mortality in acute type A aortic dissection. J Thorac Dis 2019;11(9):3887-3895. doi: 10.21037/jtd.2019.09.11 


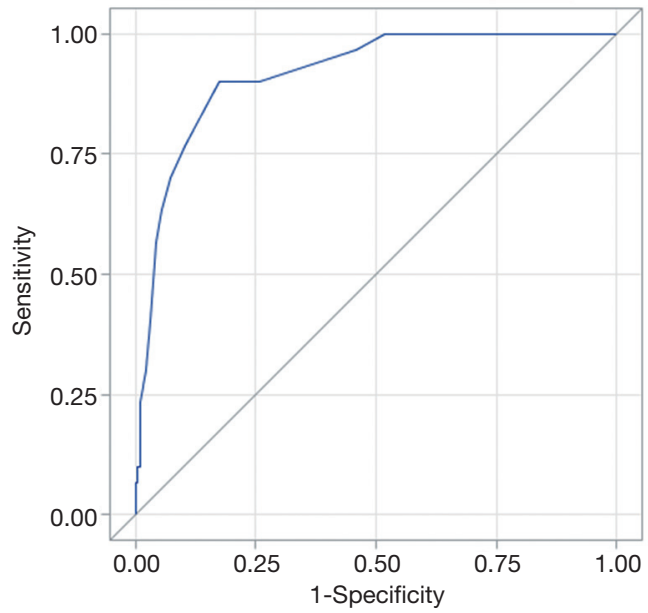

ROC curve for APACHE II score area under the Curve $=0.9172$

Figure S1 Receiver operating characteristics curve for APACHE II score in predicting TAAD in-hospital death. TAAD, type A aortic dissection. 